\title{
Scenarios in virtual learning environments for one-to-one communication skills training
}

\author{
Raja Lala ${ }^{1 *}$ (D) Johan Jeuring ${ }^{1,2}$, Jordy van Dortmont ${ }^{1}$ and Marcell van Geest ${ }^{1}$
}

\author{
*Correspondence: r.lala@uu.nl \\ 'Utrecht University, Utrecht, \\ Netherlands \\ Full list of author information is \\ available at the end of the article
}

\begin{abstract}
A scenario is a description of a series of interactions between a player and a virtual character for one-to-one communication skills training, where at each step the player is faced with a choice between statements. In this paper, we analyse the characteristics of scenarios and provide a classification to represent such scenarios. The analysis is performed through a literature review and by comparing virtual learning environments for scenario based training. Using this analysis we specify requirements for describing communication scenarios related to their: structure (linear, branching, interleaving), properties (static information stored per scenario like situation, background, which virtual character to show), and parameters (characteristics of a scenario that can be modified per statement like a score on a learning goal and an emotional effect in a virtual character). We define a schema for representing such communication scenarios and present an authoring tool to create a scenario.
\end{abstract}

\section{Introduction}

One-to-one communication skills are essential in almost all professions. A doctor Silverman, Kurtz, and Draper (2013), veterinarian Gray and Moffett (2010), and most other professionals need to appropriately communicate with clients. Many university and vocational programs require communication skills, and train their students in communication skills. A doctor, a psychologist and a veterinarian need to practice a consult with a patient or an owner. A consult varies from gathering the history of a patient to understanding the symptoms of a complaint. An engineer and an IT expert discuss system requirements with a client while a pharmacist advises a customer who comes to a pharmacy to collect medicines for the first time. More generic communication skills such as conflict management, negotiation, and behaving assertively are useful for many professionals. Communication skills are also often trained on the job, at any level: from management (how to deliver bad news), to call center operators (how to convince people to buy a particular product).

Communication skills are best learned through practice, in role-play or with a simulated patient (Berkhof, Rijssen, Schellart, Anema, \& Beek, 2011). Feedback has a positive effect in such training situations. Training one-to-one communication skills requires at least three people: two people performing a conversation, and a third assessing the conversation and skills. Such trainings require a substantial amount of time and organisation. The last decade has seen the development of a number of digital

(c) The Author(s), 2017 Open Access This article is distributed under the terms of the Creative Commons Attribution 4.0 International License (http://creativecommons.org/licenses/by/4.0/), which permits unrestricted use, distribution, and reproduction in any medium, provided you give appropriate credit to the original author(s) and the source, provide a link to the Creative Commons license, and indicate if changes were made. 
environments that support training communication skills. A student performs a conversation with a virtual character, or with a character that appears in videos, and the learning environment assesses the performance of the student. Usually these environments do not replace the traditional real-life trainings, rather they are used to better prepare students for such training sessions, or to offer training possibilities in situations where it is hard to arrange real-life training sessions, or as a complement to such trainings. As far as we are aware there does not exist an effect analysis study on the use of virtual characters in communication skills training. In general, using virtual humans in health care educational programs leads to results comparable to other training methods (and is significantly better than using no intervention) (Cook, Erwin, \& Triola, 2010).

Learning environments for training one-to-one communication skills offer scenarios that typically occur in real-world situations. A scenario consists of a setting, and a sequence of usually alternating statements between a student and a virtual character. A bad news conversation for a doctor typically involves a diagnosis of a terrible illness, for a manager firing an employee. Although these bad news conversations in different situations follow a similar pattern, the subtleties of a conversation and the responses are very different.

At an abstract level, we can compare scenarios along several axes:

- Does the scenario follow a predefined structure or not?

- What aspects of a virtual character can be controlled by a scenario: utterances, emotions, gestures, ...?

- Are both the choices for a student and the responses from a virtual character completely scripted, or are some of these aspects generated by means of some AI technique?

- Is it possible to define or use learning goals, and score interactions from a student on these goals?

Scenarios for interactive learning environments are a relatively recent phenomenon. This paper investigates the various variants of scenarios that have appeared in the literature, compares them, and proposes a structure for scenarios to have a common language to describe a scenario. A common structure for scenarios is also useful to provide a means to validate a scripted scenario between a player and a virtual character; for developing a (visual) editor for a scenario that can be used by a domain expert to develop a scenario; and to simulate a dialogue based on a scenario.

This paper is organised as follows. Section "Learning environments for communication skills" discusses virtual learning environments that offer scenario based training or assessment. Section "Characteristics of scenarios" distils the characteristics from virtual learning environments, proposes a definition for scenarios and presents an authoring tool for creating a scenario. Section "Use cases" describes various use-cases. Section "Conclusion and future work" concludes.

\section{Learning environments for communication skills}

In this section we list the virtual learning environments for communication skills we found in the literature, and describe their main characteristics. Although not our main focus, we also include environments primarily used for assessment. 
Vogel et al. (2006) define a game as software that has a goal, is interactive, and is rewarding (gives feedback). Interactive simulation activities must interact with a user by offering options to choose or define parameters of a simulation. A user then plays a newly created simulation rather than simply selecting a pre-recorded simulation.

The top-level characteristic for learning environments for communication skills is whether or not, or to what extent, scenarios are scripted. According to Realdon, Zurloni, Confalonieri, Mortillaro, and Mantovani (2006), scripting different ad hoc perspectives is a fundamental prerequisite for a narrative structure in order to reproduce both the flexibility and regularity of communicative exchanges. In 'Face-to-Face Interaction with Pedagogical Agents, Twenty Years Later,' Johnson and Lester (2016) evaluate the evolution of features of pedagogical agents over two decades. They present the possibility of interactive natural language dialogue by combining advances in natural language understanding and dialogue management. They argue that complex technologies can be difficult to understand, control, and author; and that this could stand in the way of acceptance and adoption of these technologies by teachers and other stakeholders.

Although agent based approaches for generating a scenario have made considerable progress in the last decade (Bosch, Brandenburgh, Muller, \& Heuvelink, 2012), there are still some problems to solve to achieve realistic simulations in which the degrees of freedom are suitable (Muller, Heuvelink, van den Bosch, \& Swartjes, 2012). In the latter paper, Muller et al. mention that an advantage of an agent approach is that the non-deterministic behaviour in an agent approach allows for variability in a gameexperience. As a disadvantage, they mention that developing an agent based model requires a greater up-front development effort when compared to scripts. It also requires a stricter collaboration between game designers, domain experts and programmers than with scripting approaches, as agent behaviour is not solely defined by fixed scripts. They mention the need for authoring tools to support game designers in developing content.

Communication skills teachers from our university view non-deterministic behaviour in a communication scenario as a disadvantage rather than an advantage. In teaching a communication protocol like bad news, a consultation, a negotiation, a conflict and the like, communication experts want to precisely control every utterance of a virtual character.

In this paper we restrict ourselves to learning environments in which scenarios are scripted, at least to a large extent. We do not include environments in which scenarios are generated by virtual agents approaches.

\section{Selecting relevant literature}

Learning environments for communication skills are used in very different contexts, and collecting the available (descriptions of) environments is non-trivial. We searched for papers on learning environments for communication skills on Scopus by looking at the title, abstract, and keywords using a query and refined the query stepwise.

The first step is a search for use of a scenario in communication skills. This leads to $2000+$ results in 27 subject areas ranging from Chemistry to Humanities. The first articles were published in the 1970's; there are 100+ results each year since 2006, the number of results rising steadily till 2013 to $200+$ articles each year and a slight decline in 2016 (176 articles). 
In a meta-analysis of the cognitive and motivational effects of serious games, Wouters, Van Nimwegen, Van Oostendorp, and Van Der Spek (2013) find that serious games were found to be more effective in terms of learning and retention, but not more motivating than conventional instruction methods. We refine our search to the area of serious/applied games. This leads to 668 results in 23 subject areas. The first articles date from the 1980's, and there are already ten articles in 2017.

For this paper we refine the search query to virtual environments and simulations. This leads to 19 results; the first article is from 2008 and there are fewer than 5 articles per year since. The papers are in 9 subject areas: Psychology, Earth sciences, Chemistry, Humanities, Medicine, Mathematics, Engineering, Social sciences and Computer science. Finally, we refine the search further to exclude environments that focus on agent approaches. This search leads to fourteen papers and the trend is the same as in the previous step.

The final search query is:

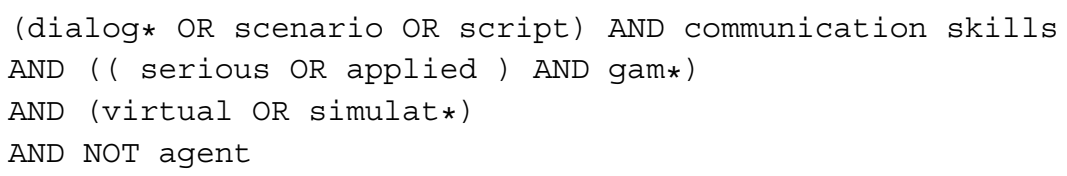

The use of scripted scenarios in virtual learning environments for communication skills training is a relatively new area used in selected domains.

Besides this query, we asked communication skills teachers from the faculties of Pharmacy, Medicine, Veterinary medicine, and Psychology at our university to provide references to research papers on learning environments for communication skills in their respective fields. After scanning the abstracts returned by our query and the suggestions from the communication skills teachers, we selected nine papers that describe a learning environment with scripted communication skills scenarios (Bosse and Provoost 2015; Bracegirdle and Chapman 2010; Cláudio et al. 2015; Gebhard et al. 2011; Guo et al. 2014; Jeuring et al. 2015; Leuski and Traum 2011; Réty et al. 2008; Wauters et al. 2012).

\section{Analysing the literature}

The selected papers use varying terms to describe similar concepts, for example: scenario or dialogue, section or subject, statement or fragment or choice. In this section, we consistently use a single term for such a concept. We describe these terms on an abstract level and go into more detail in the following paragraphs per paper.

- A scenario is a sequence of interleaved subjects in a context.

- A subject is a directed graph of statements within a scenario, usually dealing with a particular theme.

- A statement is a piece of text.

- An authoring tool is software that allows an author (usually a non-programmer) to create a scenario.

Bracegirdle et al. (2010) present a 'programmable patient', with a 'brain' that is essentially a scenario in our description. Clinical educators can add subjects to a scenario. A student asks or selects questions that correspond to nodes in a tree. If a question is not recognized, a student can choose to store the question together with an answer in this tree. Scenarios are not completely scripted: questions corresponding to nodes can be asked in various 
ways. A scenario does not affect the emotion of a programmable patient (virtual character) in this game. A programmable patient is standardised in comparison to an actor and this leads to equity in assessment.

Bosse et al. (2015) use a scenario in which a student chooses between several statements. In addition to statement choices for a student, a statement of a virtual character may also have choices. The latter choices depend on values of emotional parameters, which in turn may vary for different characters. A statement choice of a student influences the value of these parameters. The main technology in the implementation is a scenario with conditional branches, in which emotional parameters are used. Students find this game an effective learning tool.

Gebhard et al. (2011) present Visual SceneMaker, an authoring tool for creating interactive applications with multiple virtual characters. SceneMaker separates content, such as statements, from logic, such as conditional branching. Content is specified in a scenario in the form of statements and stage directions for controlling gestures, postures, and facial expressions. The logic of an interactive performance and interaction with virtual characters is controlled by a sceneflow. A sceneflow is a hierarchical scenario with nodes that structure content of an interactive presentation and edges that specify how this content is linked together. Visual SceneMaker is evaluated in experiments; the visual programming approach and modelling structures are comprehensible and let non-experts create virtual character applications in a rapid prototype fashion.

Claudio et al. (2015) demonstrate Virtual Humans (VH) in an interactive application, the goal of which is to train and assess self-medication consultation skills. A domain expert develops a scenario using an authoring tool called Dialogue Creator. A scenario is a graph implementation and consists of a sequence of statements between a $\mathrm{VH}$ patient and a player, with associated scores per answer. Each node in a graph corresponds to a statement that is articulated by a $\mathrm{VH}$ or a statement option for a player and its associated score. Seven pharmacy experts performed an evaluation of the application. These experts consider this application a valuable tool for training and assessing the over-the-counter counselling skills of a player, and recommend to follow such training with real interactions and live evaluation.

Leary's Rose (2012) focusses on natural language recognition of player sentences. Input sentences are classified on two axes: the vertical axis indicates whether a speaker is dominant or submissive towards a listener, and the horizontal axis indicates a speaker's willingness to cooperate with a listener. A scenario engine selects an appropriate response to a player's input on the basis of identified keywords and relative positions of both player and Virtual Agent on the axes. A player's input is fed to a finite state machine (FSM) and if an input matches a state, the FSM selects a reply from the available follow-up states.

Rety et al. (2008) present a framework for interactive narrative authoring in the domain of virtual storytelling where a user is provided with navigation choices in an interactive narrative. The framework is based on a generalised concept of a section, which is close to a subject in our description. A subject consists of hierarchically structured fragments (a statement in our description). A statement is defined as an atomic content element containing simple text and possibly other media. A statement may also be composed recursively and consist of a set of statements, a behaviour and a termination property. Behaviour can be set by an author as either deterministic to indicate that a user 
reads in the order of subjects or as non-deterministic to indicate that a user reads with no predefined order. A termination property is a condition that an author sets to determine when a subject terminates. A user then moves on to a following subject. A user reads an interactive narrative created in this framework by successively accessing statements in a subject.

Leuski and Traum (2011) describe a Non Player Character (NPC) editor, which includes natural language understanding and generation, and statement management. This authoring tool is designed primarily to develop question answering characters. The focus is to deliver an appropriate answer for a user's question. An author creates a response handling strategy by populating a language database with specific answers to sample questions and assigning these answers to an individual character. Different characters may have different knowledge about an event and respond differently to the same question. At run-time, a statement manager matches a user's question to an answer of an associated non player character. NPCEditor has been evaluated and is an effective and versatile system in applications for virtual question answering characters. As future work, the autors mention dialogue management as a specialized component.

Jeuring et al. (2015) describe Communicate, a serious game for practicing communication skills. An important aspect of Communicate is the de-coupling of scenario development by communication skills experts from the implementation. A communication expert iteratively develops a scenario as a directed acyclic graph of statements in an authoring tool. A statement has an incremental score, emotional effect, and feedback. Statements can be structured under subjects. A scenario parser uses this graph to generate a scenario specific reasoner. During gameplay, a player receives information about statement choices at each step in a series of interactions with a virtual character. At the end of a simulation a player receives a final score on communication skills.

Guo et al. (2014) present a game prototype for practicing communication skills in a medical consultation process. The communication skills module accesses knowledge content and a scenario through a restful web service. Knowledge is modelled as rules, which determine which player actions have a positive or a negative effect during a medical consultation, as well as the influence of each type of action on a patients stress level. A scenario models a virtual patient with some key characteristics, which are chosen from the knowledge content. A scenario also defines possible actions for a player with a virtual patient in each phase of a medical consultation process.

\section{Dialogue authoring assets}

In addition to the literature review, we looked at available dialogue authoring tools by searching in the Unity asset store (www.assetstore.unity3d.com) and asking game developers from the RAGE (Realising an Applied Gaming Eco-system: www.rageproject.eu) project for recommendations. From the selection, we looked at:

- Dialoguer (www.dialoguer.info)

- Dialogue System (www.pixelcrushers.com)

- Dialogue (www.ninjapokestudios.co.uk) 
- Articy (www.nevigo.com)

- Chatmapper (www.chatmapper.com)

- Twine (twinery.org)

These authoring tools concentrate mostly on the dialogue aspect of a scenario used in entertainment games. They range in functionality from simple tools offering basic dialogue sequences to advanced tools. We identify three features relevant to a scenario in these tools.

- The tools support the construction of a sequence of statements representing a dialogue flow from a player to a computer and vice-versa.

- They provide the possibility for a player to have multiple choices as a response to a computer question or a situation.

- Many tools use preconditions; a particular dialogue fragment is only reached if a certain condition is fulfilled. For example, a player must have completed a certain task prior to reaching a particular dialogue fragment.

Articy is a visual environment for the creation and organization of game content offering virtual characters, locations and a game development environment. Chatmapper has a basic free version available. Twine is an open-source tool for creating interactive, non-linear stories and resembles Interactive narratives (Réty et al. 2008).

\section{Characteristics of scenarios}

In this section, we extend the description of a scenario given in the previous section to more precisely define what we mean with a scenario. We formalise this definition in an XML schema, which we can use to validate a scenario. We present a scenario editor using which a scenario author can create a scenario.

\section{Scenario definition}

The majority of the papers in the literature (Bosse and Provoost 2015; Bracegirdle and Chapman 2010; Cláudio et al. 2015; Gebhard et al. 2011; Jeuring et al. 2015; Réty et al. 2008) describe a communication exchange between a player and a virtual character as a tree, graph or chart. Communication flows from a virtual character to a player and viceversa. Player and virtual character statements are represented as nodes and the flow of conversation is represented as an edge with a direction. A dialogue proceeds from one statement to another between characters (player and virtual) and flows forward. Such a communication exchange can be represented by a directed acyclic graph (DAG). A DAG consists of a finite set of nodes and edges between nodes, with no cycles. We define a communication exchange, often dealing with a particular subject, as a DAG. Characteristics of a subject DAG, as found in commercially available dialogue authoring assets/tools and in the literature (Bosse and Provoost 2015; Cláudio et al. 2015; Jeuring et al. 2015; Réty et al. 2008), are:

- multiple player nodes to represent player statement choices.

- a precondition in a node.

Bosse et al. (2015) present a game for aggression de-escalation training with a scenario simulation in a public transport tram. Bracegirdle et al. (2010) and Guo et al. (2014) 
present simulations in clinical communication skills which play in a hospital and a clinic respectively. Claudio et al. (2015) present a game which plays in a pharmacy and allows student engagement in a true-to-life situation in a controllable environment. Jeuring et al. (2015) present a simulation where a teacher can select from virtual characters and backgrounds in a context of a scenario. According to Realdon et al. (2006), an adequate scaffolding structure for the training of communicative competence in an e-learning environment entails fixing both contextual boundaries and degrees of freedom to let the learner have the opportunity to give sense to the perspective selected. We infer that a communication scenario has a context in which a student/user plays. Three possible components of a context are for example a location, a background image and a hair color for an interactive virtual character. We define these components as properties for a scenario that provide context during gameplay. A property is a static piece of information for a scenario, a statement or a virtual character. An example of a property for an entire scenario is a location, of a property for a statement is an intent of a player, and of a property for a virtual character is a name.

Since we search for literature on serious or applied games, most papers (Bosse and Provoost 2015; Bracegirdle and Chapman 2010; Cláudio et al. 2015; Gebhard et al. 2011; Guo et al. 2014; Jeuring et al. 2015; Wauters et al. 2012) mention learning goals. A player's choices determine his/her score on these learning goals. A player can get feedback either during gameplay following a player choice or at the end of a scenario. In some of the games (Bosse and Provoost 2015; Jeuring et al. 2015; Wauters et al. 2012) an emotional state of a virtual character can change depending on a player statement choice. In papers that describe an authoring tool for scenarios (Bosse and Provoost 2015; Gebhard et al. 2011; Jeuring et al. 2015; Leuski and Traum 2011; Réty et al. 2008), a scenario writer may change such an emotional state or progress towards a learning goal by defining an effect on a parameter at a statement. We define a parameter as a dynamic piece of information of a scenario that can be modified per statement. Examples of parameters are a character's emotion and a score for a scenario.

In interactive narratives (Réty et al. 2008) a user may read subjects in a hierarchy without any predefined order. To overcome predictable behaviour in a conversation with an agent, agents in conversational trees (Bosse and Provoost 2015) are endowed with an internal state to introduce variability in statements. In Communicate! (Jeuring et al. 2015), interleaving enhances expressiveness in a communication scenario by allowing students to navigate a conversation with a virtual character through subjects. If the order in which statements within two subjects (or more) are navigated is not important, then these subjects are interleaved. We infer that variability in navigating subjects in a scenario is important to add expressiveness to a scenario and partly overcomes predictable behaviour. We characterise this variability for two (or more) subjects as interleaved in a scenario where a player gets statement choices from within these interleaving subjects without a predetermined order. We define a scenario as a sequence of interleaved subjects.

Summarising, in our definition, a scenario is a sequence of interleaved subjects, where each subject is a DAG. In a scenario static information is represented by properties, and dynamic information by parameters. An overview of which concepts appear where in the selected papers and tools is depicted in Table 1. 
Table 1 Which concepts are used where?

\begin{tabular}{lllll}
\hline Papers & Scenario structure & Parameter & Property & Dialogue variability \\
\hline Bosse et al. & $\checkmark$ & $\checkmark$ & $\checkmark$ & $\checkmark$ \\
Bracegirdle et al. & $\checkmark$ & $\checkmark$ & $\checkmark$ & \\
Claudio et al. & $\checkmark$ & $\checkmark$ & $\checkmark$ & $\checkmark$ \\
Gebhardt et al. & $\checkmark$ & $\checkmark$ & $\checkmark$ & $\checkmark$ \\
Guo et al. & & $\checkmark$ & $\checkmark$ & \\
Jeuring et al. & $\checkmark$ & $\checkmark$ & $\checkmark$ & $\checkmark$ \\
Leuski et al. & $\checkmark$ & $\checkmark$ & & $\checkmark$ \\
Realdon et al. & $\checkmark$ & & & \\
Rety et al. & $\checkmark$ & & & \\
Vaasen et al. & & $\checkmark$ & & \\
Tools & & & & \\
Dialoguer & $\checkmark$ & $\checkmark$ & & \\
Dialogue system & $\checkmark$ & $\checkmark$ & \\
Dialogue & $\checkmark$ & & \\
Articy & $\checkmark$ & $\checkmark$ & \\
Chatmapper & $\checkmark$ & $\checkmark$ & \\
Twine & $\checkmark$ & & \\
\hline
\end{tabular}

XML schema for validating a scenario

We define the Utrecht University Dialogue Scenario Language (UUDSL) as an XML language for describing the structure of a scenario including a dialogue structure, virtual character(s), properties (e.g. location, character name) and parameters (e.g. emotion, score).

It is schematically represented in Fig. 1

The top-level element contains definitions, metadata and an element for sequencing interleaved subjects. Characters, properties and parameters are defined inside the definitions element for reference in the rest of the dialogue scenario. A property is a static piece of information with a scope. A property scope can be per-statement or independent of statements, which evaluates to a piece of information about a scenario. A parameter is, in contrast to a property, a dynamic piece of information and is by definition per-statement. A statement can change the value of a parameter. A character has an ID and a set of properties and parameters.

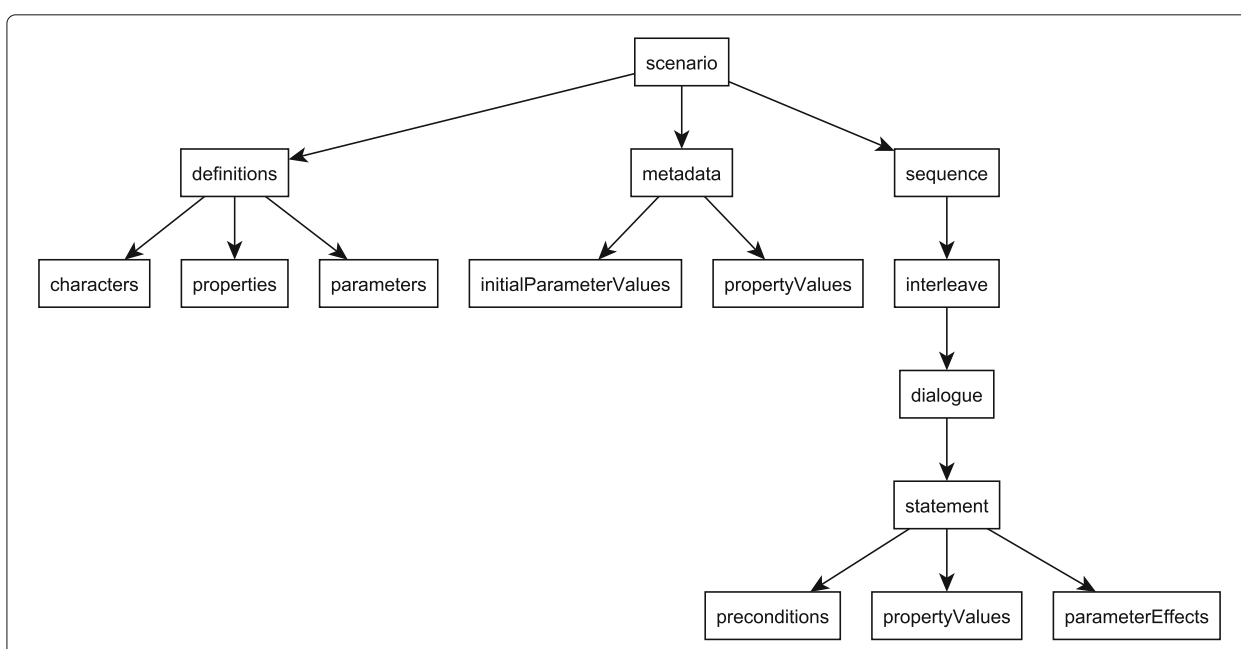

Fig. 1 Schematic representation of the UUDSL 
The metadata element contains all data that describes a scenario, but is not part of it (e.g. name, description). The metadata element also contains property values and initial parameter values. These initial parameter values are used to initialise a scenario's parameters during game-play. Property values stored in metadata define properties of a scenario.

The sequence element contains a sequence of interleaved subjects. The interleave element delimits the interleaving dialogue elements, which represent a piece of dialogue about a subject. Inside a dialogue element there are statements representing the contents and effects of the dialogue. A statement element contains text, preconditions, property values, parameter effects, links to possible response statements and whether the statement belongs to a player or computer.

The UUDSL can be accessed at: https://uudsl.github.io/scenario/.

\section{An authoring tool for creating a scenario}

In this section we present a scenario editor, an authoring tool in which a communication expert (an author) iteratively develops a communication scenario as a sequence of interleaved subjects, consisting of statements with parameters per step. This editor has been developed in collaboration with communication teachers from our university and external users, and runs in a web-browser.

The editor has the following basic features:

- Dialogues are structured in subjects.

- An author develops a subject in a scenario as a DAG of statements between a virtual character and a player. The subject represented by a DAG starts at the top and ends at the bottom.

- Within a subject, an author develops a dialogue between a virtual character (computer node) and a player (player node).

- An edge from a computer node to a player node (and vice-versa) depicts a flow of conversation.

- An author connects a computer node to multiple player nodes to create statement choices for a player.

- Each player statement choice leads to an incremental score, and affects for example an emotion in a virtual character.

- A statement can have a precondition.

Figure 2 shows an example scenario, which consists of 5 subjects.

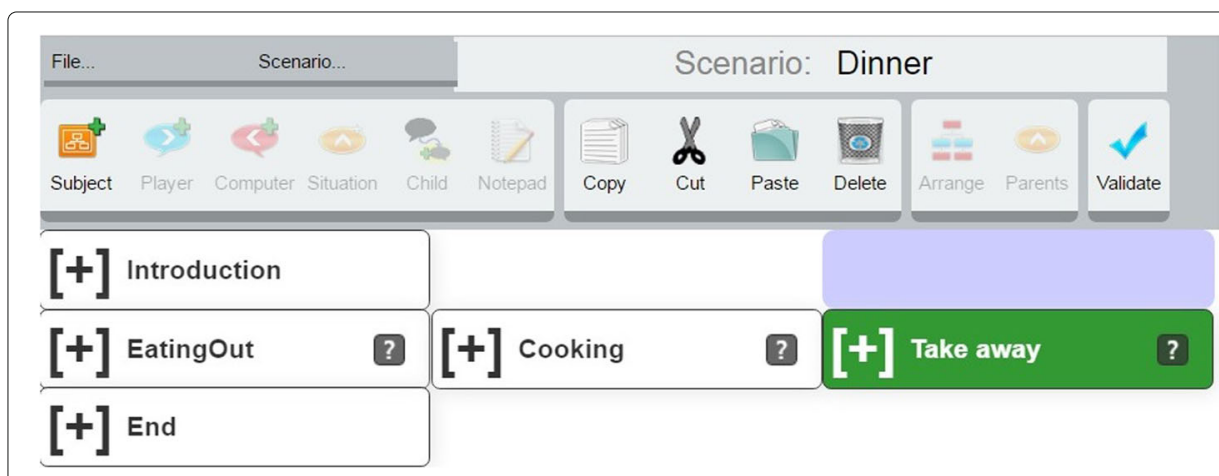

Fig. 2 Example scenario subject level 
Subjects on the same horizontal level are interleaved. In Fig. 2, 'EatingOut', 'Cooking', and 'Take away' are interleaved subjects. This indicates that a player will get statement choices from within these subjects in no particular order during a simulation of this scenario. She can navigate these subjects in any order in a simulation.

A subject may be marked as optional, depicted in Fig. 2 with a question-mark, which means that the subject can be skipped altogether in a simulation.

Figure 3 below shows an example of a DAG for a subject (in this example the 'EatingOut' subject from Fig. 2).

The scenario editor is configurable: a game developer can specify a virtual character(s), properties (e.g. name of a character) and parameters (e.g. emotion and gesture) specific to a game. We provide a configuration XML schema to create a game-specific instance of the authoring tool. A scenario author, usually a non-programming expert, can use this instance of the scenario editor to create a scenario(s) specific to a game. The resulting scenarios and their associated properties and parameters can be used by the game developer for the game.

In an example configuration, the type of a property such as location can be specified by a game developer as an enumeration with the elements town, village and city. This enables a scenario author to select a location from the enumeration as a context for a scenario. The name property of a virtual character can be specified by a game developer as a value of the type string, which allows a scenario author to define the name of the character. The emotion for a virtual character can be specified by a game developer as an enumeration parameter consisting of elements angry, happy and sad. This parameter enables a scenario author to create an effect on the emotion of a virtual character at a statement. Additionally, a scenario author can also define a user parameter for a specific

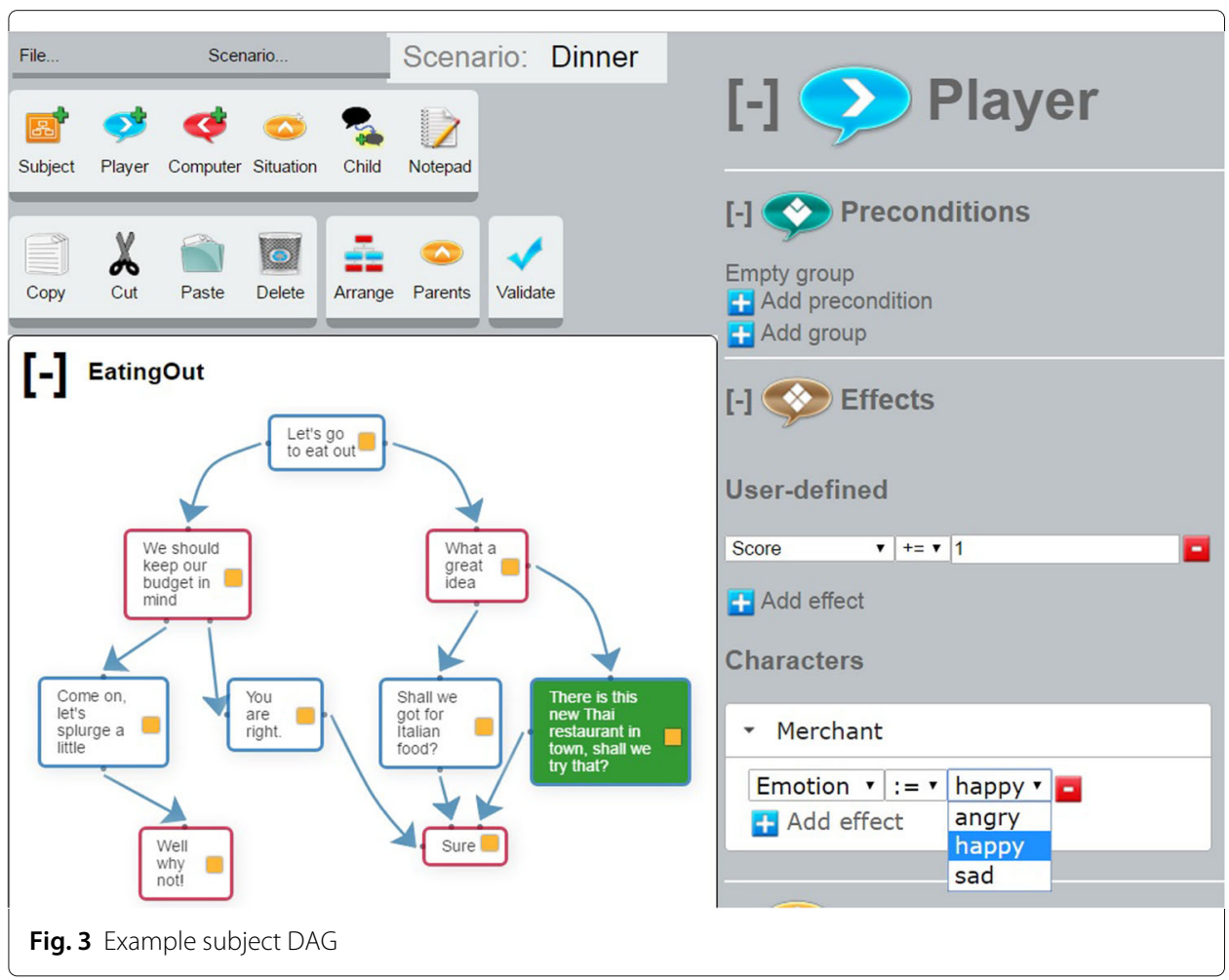


scenario, for instance a score as an integer, which can be set at a player statement. Figure 3 also displays the parameters of this example configuration of the authoring tool.

Summarising, our authoring tool:

- is developed from a communication teacher's (non-programmer) perspective. Existing tools (reviewed in Section "Dialogue authoring assets") are primarily developed for game-developers (programmers). Our tool leads to de-coupling of scenario content from programming and a domain expert can develop and modify a scenario independent of a game developer.

- runs in a web browser as opposed to existing client-based tools. This makes it more easily accessible to scenario authors.

- is (easily) configurable to the needs of a scenario author or a game-developer.

- creates a scenario that can be validated by using a schema.

- develops a scenario as a sequence of interleaved subjects. This abstraction of a scenario (often following a communication protocol) in subjects is unique.

Our authoring tool is open-source and can be accessed at: https:/github.com/ UURAGE/ScenarioEditor.

An author can export a scenario from the authoring tool. The exported scenario is an XML document based on the UUDSL and can be validated using the UUDSL Schema. An exported scenario XML is integrated in a game.

\section{Use cases}

This section describes how our UUDSL and authoring tool can be used in various applications.

Our authoring tool is used with a serious game as part of a learning environment for practising communication-skills (https://communicategame.nl/) at our University. This environment is used by over 20 communication teachers and teaching-assistants from the faculties of Psychology, Pharmacy, Medicine and Veterinary science as part of their curriculum to teach communication skills to their respective students. This environment is also used by a training company, who trains clients in communication skills. An example client is a foundation that trains healthcare professionals working in poor neighbourhoods.

We work in a broader context in the RAGE (Realising an Applied Gaming Eco-system) project (www.rageproject.eu). RAGE brings together three kinds of stakeholders: game asset developers such as universities and research institutes; game companies who integrate game assets to form an applied game, and users of applied games, universities, colleges or employment and training agencies who wish to deploy applied games in education and training of students or personnel. Our editor is one of the RAGE assets.

Our editor asset is used by one of the RAGE pilot games. In this game, a player works in a virtual game studio as an office assistant. Her goal is to increase the success of the virtual company by improving the social skills of a virtual team. During game-play, a player can visit a water cooler in the virtual company to converse with different virtual characters. A dialogue starts with a statement from a virtual character for group working/conflict management behaviour and a player can choose to reply from multiple-choice statements. A teacher/communication expert from the college scripts these dialogues using a 
configured instance of our editor asset. The college uses a pedagogical model based on the Thomas-Kilmann Conflict Mode Instrument (Kilmann and Thomas 1975). A dialogue requires a player statement choice to have an intent. This intent is configured as a property of a statement with values: Competing, Accommodating, Avoiding, Collaborating or Compromising. A game development company integrates these dialogues in a game. The game will be piloted and evaluated by students.

Another RAGE asset is a virtual human controller that uses BML (Behavior markup language) input to control aspects of a virtual character, for instance face emotion and arm gesture. We configured an instance of our editor to specify an emotion and a gesture for a virtual character. Both are defined as a parameter of type enumeration. We integrated our assets in a simple Unity game that plays a scenario from our asset with a virtual character from the other asset. Initially, the other asset supported three emotions: happy, angry and sad. Later 'surprised' was added and it was easy to add an element to the enumeration parameter in our editor. We can modify and play a scenario without changing the software of the game.

We analyse how our authoring tool can be used for some of the games described in the reviewed papers. We sketch a configuration of the scenario editor to enable a scenario author to create a scenario for a game in the reviewed papers. For Bosse et al. (2015), the editor configuration consists of a single virtual character; parameters: a score (integer) and feedback to a player (string); properties: a context (a string) with value aggression deescalation, and a background-image with as value the image of a tram. For the pharmacy communication simulation of Claudio et al. (2015), the editor instance has a single virtual character, a scoring (integer) parameter, and a background-image property with as value a picture of a clinic. Finally in the simulation from Guo et al. (2014), the editor instance offers a single virtual character, a background-image property with as value a picture of a medical consultation room, and a scoring parameter.

\section{Conclusion and future work}

Communication skills are needed in the majority of professions and are best learned through practice. These skills may be developed in learning environments that simulate real-world communication scenarios, for instance a bad news conversation. We analyse the literature and contemporary simulations and tools for common characteristics of communication scenarios. Characteristics of communication scenarios are their structure, properties, and parameters. We propose a schema that models the various aspects of communication scenarios. We present a configurable authoring tool to create a scenario. We can use this tool to construct scenarios for various applied games.

RAGE aims to deliver five pilots of applied games. Our asset is also planned to be used in two other games; one intended for a job-seeker to practice interview skills at an employment agency and another for a student to practice a business negotiation. In the context of Rage, we plan to investigate hybrid approaches to dialogue management in applied games together with INESC (Dias et al. 2014). In our methodology, we handle dialogue management in a scripted, centralised manner. In a distributed agent approach, a dialogue emerges from individual decisions that are made by a virtual character according to their internal Artificial Intelligence. We can experiment if a hybrid approach leads to more variability during game-play while preserving the consistency and other 
advantages of a scripted approach as described in Section "Learning environments for communication skills".

We also want to determine schema extensions that enable us to translate a high-level communication protocol to communication scenarios. An example of such a protocol is the protocol to hand out medicine to a customer who visits a pharmacy for the first time. A scenario based on this protocol varies depending on aspects such as the side-effects of the medicine, and the name of a character. Another example of a protocol is a bad news conversation, which for a doctor involves telling a patient about a diagnosis of a terrible illness, for a veterinarian telling an owner of a pet about a diagnosis, and for a manager firing an employee. It is a research question how we best can pass arguments to communication protocols.

\section{Acknowledgements}

This work has been partially funded by the EU H2020 project RAGE (Realising an Applied Gaming Eco-system, http:// www.rageproject.eu/), grant agreement no 644187. This paper reflects only the author's view, the European Commission is not responsible for any use that may be made of the information it contains.

\section{Publisher's Note}

Springer Nature remains neutral with regard to jurisdictional claims in published maps and institutional affiliations.

\section{Author details}

${ }^{1}$ Utrecht University, Utrecht, Netherlands. ${ }^{2}$ Faculty of Management, Science and Technology, Open University of the Netherlands, Heerlen, Netherlands.

Received: 14 November 2016 Accepted: 3 April 2017

Published online: 11 May 2017

\section{References}

Berkhof, M., Rijssen, H.J.V., Schellart, A.J.M., Anema, J.R., \& Beek, A.J.V.D. (2011). Effective training strategies for teaching communication skills to physicians: An overview of systematic reviews. Patient Education and Counseling, 84(2), $152-162$.

Bosch, K., Brandenburgh, A., Muller, T.J., \& Heuvelink, A. (2012). Characters with personality! In Proceedings IVA 2012: the 12th International Conference on Intelligent Virtual Agents. LNAI, vol. 7502 (pp. 426-439). Springer.

Bosse, T., \& Provoost, S. (2015). Integrating conversation trees and cognitive models within an eca for aggression de-escalation training. In Proceedings PRIMA 2015: the 18th International Conference on Principles and Practice of Multi-Agent Systems. LNCS, vol. 9387 (pp. 650-659). Springer.

Bracegirdle, L., \& Chapman, S. (2010). Programmable patients: Simulation of consultation skills in a virtual environment. Bio-algorithms \& Med-Systems, 6, 111-115.

Cláudio, A.P., Carmo, M.B., Pinto, V., \& Cavaco, A. (2015). Virtual humans for training and assessment of self-medication consultation skills in pharmacy students. In Proceedings ICCSE 2015: the 10th International Conference on Computer Science \& Education (pp. 175-180). IEEE.

Cook, D.A., Erwin, P.J., \& Triola, M.M. (2010). Computerized virtual patients in health professions education: a systematic review and meta-analysis. Academic Medicine, 85(10), 1589-1602.

Dias, J., Mascarenhas, S., \& Paiva, A. (2014). Fatima modular: Towards an agent architecture with a generic appraisal framework. In Emotion Modeling: Towards Pragmatic Computational Models of Affective Processes, LNCS, 8750 (pp. 44-56). Springer.

Gebhard, P., Mehlmann, G., \& Kipp, M. (2011). Visual scenemaker-a tool for authoring interactive virtual characters. Journal on Multimodal User Interfaces, 6(1), 3-11. doi:10.1007/s12193-011-0077-1.

Gray, C., \& Moffett, J. (2010). Handbook of Veterinary Communication Skills. Wiley-Blackwell.

Guo, J., Singer, N., \& Bastide, R. (2014). Design of a serious game in training non-clinical skills for professionals in health care area. In Proceeding SEGAH 2014: IEEE International Conference on Serious Games and Applications for Health. http://oatao.univ-toulouse.fr/13098/ (pp. 1-6). IEEE.

Jeuring, J., Grosfeld, F., Heeren, B., Hulsbergen, M., IJntema, R., Jonker, V., ... van Zeijts, H. (2015). Communicate! — a serious game for communication skills —. In Proceedings EC-TEL 2015: Design for Teaching and Learning in a Networked World: 10th European Conference on Technology Enhanced Learning. LNCS, vol. 9307. doi:10.1007/978-3-319-24258-3_49. http://dx.doi.org/10.1007/978-3-319-24258-3_49. (pp. 513-517). Springer.

Johnson, W.L., \& Lester, J.C. (2016). Face-to-face interaction with pedagogical agents, twenty years later. International Journal of Artificial Intelligence in Education, 26(1), 25-36. doi:10.1007/s40593-015-0065-9.

Kilmann, R. H., \& Thomas, K. W. (1975). Interpersonal Conflict-Handling Behavior. Psychological Reports, 37(3), 971-980.

Leuski, A., \& Traum, D. (2011). NPCEditor: Creating Virtual Human Dialogue Using Information Retrieval Techniques. Al Magazine, 32(2), 42-56.

Muller, T.J., Heuvelink, A., van den Bosch, K., \& Swartjes, I. (2012). Glengarry Glen Ross: Using BDI for Sales Game Dialogues. In Proceedings AllDE 2012: 8th AAAI Conference on Artificial Intelligence and Interactive Digital Entertainment (pp. 167-172). AAAI Press. 
Realdon, O., Zurloni, V., Confalonieri, L., Mortillaro, M., \& Mantovani, F. (2006). Learning communication skills through computer-based interactive simulations. In From Communication to Presence: Cognition, Emotions and Culture Towards the Ultimate Communicative Experience (pp. 281-303). Amsterdam. IOS Press.

Réty, J.H., Szilas, N., Clément, J., \& Bouchardon, S. (2008). Authoring interactive narratives with hypersections. In Proceedings of the 3rd International Conference on Digital Interactive Media in Entertainment and Arts (pp. 393-400). ACM.

Silverman, J., Kurtz, S., \& Draper, J. (2013). Skills For Communicating With Patients, Third Edition. Radcliffe Publishing Limited.

Vogel, J.J., Vogel, D.S., Cannon-Bowers, J., Bowers, C.A., Muse, K., \& Wright, M. (2006). Computer gaming and interactive simulations for learning: A meta-analysis. Journal of Educational Computing Research, 34(3), 229-243. doi:10.2190/FLHV-K4WA-WPVQ-HOYM.

Wauters, J., Broeckhoven, F., Overveldt, M., Eneman, K., Vaassen, F., \& Daelemans, W. (2012). delearyous: An interactive application for interpersonal communication training. In Serious Games: The Challenge: Joint Conference of the Interdisciplinary Research Group on Technology, Education, and Communication, and the Scientific Network on Critical and Flexible Thinking Ghent. Communications in Computer and Information Science, vol. 280 (pp. 87-90). Springer.

Wouters, P., Van Nimwegen, C., Van Oostendorp, H., \& Van Der Spek, E. D. (2013). A meta-analysis of the cognitive and motivational effects of serious games. Journal of Educational Psychology, 105(2), 249-265.

\section{Submit your manuscript to a SpringerOpen ${ }^{\circ}$ journal and benefit from:}

- Convenient online submission

- Rigorous peer review

- Immediate publication on acceptance

- Open access: articles freely available online

- High visibility within the field

- Retaining the copyright to your article

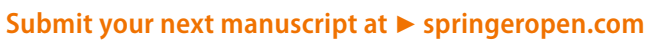

